\title{
Binge Alcohol Is More Injurious to Liver in Female than in Male Rats: Histopathological, Pharmacologic, and Epigenetic Profiles
}

\author{
Shivendra D. Shukla, Ricardo Restrepo, Annayya R. Aroor, Xuanyou Liu, Robert W. Lim, \\ Jacob D. Franke, David A. Ford, and Ronald J. Korthuis \\ Department of Medical Pharmacology and Physiology, School of Medicine, University of Missouri, Columbia (S.D.S., R.R., \\ A.R.A., X.L., R.W.L., R.J.K.), and Department of Biochemistry and Molecular Biology and Center for Cardiovascular Research, \\ Saint Louis University, St. Louis (J.D.F., D.A.F.), Missouri
}

Received April 11, 2019; accepted June 19, 2019

\begin{abstract}
Binge alcohol consumption is a health problem, but differences between the sexes remain poorly defined. We have examined the in vivo effects of three acute, repeat binge alcohol administration on the liver in male and female rats. Sprague-Dawley rats were gavaged with alcohol $(5 \mathrm{~g} / \mathrm{kg}$ body weight) three times at 12 -hour intervals. Blood and liver tissues were collected 4 hours after the last binge ethanol. Subsequently, several variables were analyzed. Compared with male rats, females had higher levels of blood alcohol, alanine aminotransferase, and triglycerides. Liver histology showed increased lipid vesicles that were larger in females. Protein levels of liver cytochrome P4502E1 were higher in the liver of females than in the liver of males after binge. Hepatic phospho-extracellular signal-regulated kinase 1/2 and phosph-p38 mitogen-activated protein kinase levels were lower in females compared with males after binge alcohol, but no differences were found in the phospho-C-jun $\mathrm{N}$-terminal kinase levels. Peroxisome proliferator-activated receptor $\gamma$-coactivator $1 \alpha$ and cyclic AMP response element binding (CREB) protein
\end{abstract}

levels increased more in female than in male livers; however, increases in phospho-CREB levels were lower in females. Remarkably, c-fos was reduced substantially in the livers of females, but no differences in c-myc protein were found. Binge ethanol caused elevation in acetylated (H3AcK9) and phosphoacetylated (H3AcK9PS10) histone H3 in both sexes but without any difference. Binge alcohol caused differential alterations in the levels of various species of phosphatidylethanol and a larger increase in the diacylglycerol kinase- $\alpha$ protein levels in the liver of female rats compared with male rats. These data demonstrate, for the first time, similarities and differences in the sex-specific responses to repeat binge alcohol leading to an increased susceptibility of female rats to have liver injury in vivo.

\section{SIGNIFICANCE STATEMENT}

This study examines the molecular responses of male and female rat livers to acute binge alcohol in vivo and demonstrates significant differences in the susceptibility between sexes.

\section{Introduction}

Alcohol consumption is a major cause of chronic liver disease in the United States and around the globe (Axley et al., 2019). Development of fatty liver disease is seen in more than $90 \%$ of alcoholics, but only $20 \%-40 \%$ of alcoholics develop further liver injury characterized by hepatitis and fibrosis (Kourkoumpetis and Sood, 2019; Taylor and Miloh, 2019). Among the factors contributing to increased susceptibility to alcohol, sex is considered an important factor contributing to increased alcoholic liver injury. Females are more susceptible to alcoholic liver injury than males are, both in clinical studies and preclinical models (Frezza et al., 1990; Becker et al.,

S.D.S. was supported by the Margaret Proctor Mulligan Endowment Fund and R.J.K. from the US National Institutes of Health National Institute of Alcohol Abuse and Alcoholism (Grant AA-022108).

https://doi.org/10.1124/jpet.119.258871.
1996; Iimuro et al., 1997; Kono et al., 2000; Yin et al., 2000; Sato et al., 2001; Fulham and Mandrekar, 2016).

In addition to sex differences, binge pattern of alcohol drinking is emerging as a significant determinant of alcoholic liver injury (Shukla et al., 2013a; Ghosh Dastidar et al., 2018). Binge drinking by humans is on the rise in many countries. More than $90 \%$ of US adults who drink excessively report binge drinking in the past 30 days. About $17 \%$ of US adults binge drink four times a month. There is epidemiologic evidence that women are more sensitive to alcohol and experience alcoholic liver injury at lowers levels of drinking than men, and for this reason the National Institute of Alcohol Abuse and Alcoholism defines binge drinking as about five or more drinks in men and about four or more drinks in women in a span of 4 hours (see White et al., 2018). Recent studies have reported increased incidence of binge drinking in women, underscoring the significance of understanding sex differences

ABBREVIATIONS: ALT, alanine aminotransferase; BAC, blood alcohol concentration; CREB, cyclic AMP response element binding protein; CYP2E1, cytochrome P4502E1; DGK $\alpha$, diacylglycerol kinase $\alpha$; ERK, extracellular signal-regulated kinase; ESI, electrospray ionization; H3AcK9, histone $\mathrm{H} 3$ acetylated at Lys 9; H3AcK9PS10, histone H3 acetylated at lys9, phosphorylated at ser10; H3PS10, histone H3 phosphorylated at Ser10; H3PS28, histone H3 phosphorylated at ser28; JNK, C-jun N-terminal kinase; MAPK, mitogen-activated protein kinase; MS, mass spectrometry; PA, phosphatidic acid; PEth, phosphatidylethanol; PGC1 $\alpha$, peroxisome proliferator-activated receptor $\gamma$-coactivator $1 \alpha$; TBST, Trisbuffered saline. 
in alcoholic liver injury (Breslow et al., 2017; Kirpich et al., 2017; Fernandes et al., 2018). Despite these differences, the pharmacologic mechanisms of the acute binge effect of alcohol on the liver of men and women have not been examined. We and others have shown changes in mitogen-activated protein kinase (MAPK) signaling components (Aroor and Shukla, 2004), alterations in transcriptional factors, and epigenetic histone modifications (Shukla and Aroor, 2006; Shukla and Zakhari, 2013b) associated with binge mode of alcohol intake (Shukla et al., 2013a) in male rat (Aroor et al., 2010, 2011) and mouse models (Shukla et al., 2015b) of alcoholic liver injury (Kim and Shukla, 2006; Bardag-Gorce et al., 2010; Aroor et al., 2012b, 2014; Kirpich et al., 2012; Abdelmegeed et al., 2013; Shukla et al., 2015b). We have used here an animal model that mimics the human acute binge scenario wherein three repeat doses of binge alcohol were administered orally, and their effects were compared in male and female rats.

\section{Materials and Methods}

All chemicals and reagents used were of the highest analytical grade available. Cell Signaling Technology (Danvers, MA) supplied antibodies for cyclic AMP response element binding protein (CREB) protein (cat. no. 9192), phospho-CREB (cat. no. 9198B), ERK protein (cat. no. 4595), phospho-ERK (cat. no. 9101), p38 protein (cat. no. 9212), phospho-p38 (cat. no. 4511), C-jun N-terminal kinase (JNK) protein (cat. no. no. 9552), phospho-JNK (cat. no. 9255), c-fos (cat. no. 2250), and c-caspase 3 (cat. no. 9961). Antibodies for c-MYC (cat. no. sc764), catalase (cat. no. sc34280), and PGC1 $\alpha$, peroxisome proliferatoractivated receptor $\gamma$-coactivator $1 \alpha$ (PGC1 $\alpha$; cat. no. sc13067) were acquired from Santa Cruz Biotechnology (Dallas, TX). Diacylglycerol kinase $(\alpha \mathrm{DGK} \alpha)$ antibody (cat. no. PA5-44058) was purchased from Thermo Fisher Scientific. CYP2E1 antibody (cat. no. ab28146) was from Abcam (Cambridge, MA). Antibodies for histone H3 AcK9 (cat. no. 06-942), H3PS10 (cat. no. 05-817), and H3PS28 (cat no. 07-145) were from Upstate Biotechnology (Lake Placid, NY). Antibody for histone H3AcK9PS10 (cat no. 9101) was from Cell Signaling Technology.

Animals and Binge Alcohol Administration. Male and female Sprague-Dawley rats were purchased from Harlan Laboratories (Indianapolis, IN). When procured, they were 7 weeks old and weighed between 250 and $300 \mathrm{~g}$. They were housed under a 12-hour light/dark cycle and were permitted ad libitum consumption of standard laboratory rat chow and water. The animal care and protocol for their use were approved (protocol no. 9092) by the University of Missouri Animal Care \& Use Committee. Rats were used for binge alcohol studies after a 1-week equilibration. We selected three-binge mode of ethanol administration, a model of repeat binge administration that is commonly seen in alcohol abusers and binge drinkers (Aroor et al., 2014; White et al., 2018). For binge ethanol administration, ethanol was diluted to $32 \%(\mathrm{v} / \mathrm{v})$ in sterile water. Using an 18-gauge stainless steel blunt-tipped needle, an average volume of $7.5 \mathrm{ml}$ of ethanol ( $5 \mathrm{~g} / \mathrm{kg}$ body weight) was administered by intragastric administration. Ethanol was administered three times at 12 -hour intervals. Water replaced ethanol in the control group. Four hours after the last binge ethanol administration, blood samples were collected, and liver was rapidly perfused with cold phosphate-buffered saline containing phosphatase inhibitors. A small portion of the liver tissue was fixed in formalin, and remaining liver tissue was quickly frozen in liquid nitrogen and stored at $-70^{\circ} \mathrm{C}$ for further analysis. We used four male and four female rats for the treatments, and data are presented as male control, male ethanol binge, female control, and female ethanol binge.

The alcohol treatment model used here has been applicable to both rats (Aroor et al., 2011, 2014) and mice (Shukla et al., 2015a). The National Institute for Alcohol Abuse and Alcoholism definition of binge refers to humans; mimicking that situation of heavy binge in small animal models is challenging and requires appropriate modifications. In the rat model used here, the blood alcohol concentrations (BACs) were in the range of $50-100 \mathrm{mM}$ (i.e., about $0.25 \%-0.5 \%$ ). This range of alcohol has been observed in human chronic alcohol abusers taking binge alcohol [discussed in Shukla et al. (2013a) and references cited therein]. Catabolism of ethanol is up to five times greater in rodents, and thus BAC levels cannot be directly compared with that of humans. The ethanol dose used here is a commonly used dose for the rat model subjected to binge in many other laboratories. Rats were still conscious and responsive to toe and tail pinch.

Determination of ALT Levels. Serum ALT levels were measured by kinetic ALT assay in an automated analyzer (University of Missouri Research Animal Diagnostic Laboratory, Columbia, MO) that we have used previously (Aroor et al., 2011).

Determination of BAC Levels. BAC levels were determined by an alcohol dehydrogenase assay kit from Genzyme Diagnostics (Framingham, MA). Serum $(100 \mu \mathrm{l})$ was used for the analysis.

Preparation of Cell Extracts and Western Immunoblot. The whole-cell extracts were prepared at $4^{\circ} \mathrm{C}$ as described (Aroor et al., 2014; Shukla et al., 2015a). After homogenizing the frozen liver tissue in lysis buffer (containing $50 \mathrm{mM}$ Tris $\mathrm{HCl}, \mathrm{pH} 7.4,25 \mathrm{mM} \mathrm{KCl}, 5 \mathrm{mM}$ $\mathrm{MgCl} 2,5 \mathrm{mM}$ glycerophosphate, $1 \mathrm{mM}$ EDTA, $1 \mathrm{mM}$ Na-ortho vanadate, $1 \mathrm{mM}$ EGTA, $1 \mathrm{mM}$ DTT, and Sigma protease inhibitor cocktail p8340), a small portion of the extract was saved. Bio-Rad DC protein assay (Bio-Rad Laboratories, Hercules, CA) was used to determine the protein concentrations in the whole cell extracts.

The whole-liver lysate protein $(40 \mu \mathrm{g})$ was separated by $10 \%$ or $15 \%$ SDS-PAGE, followed by transfer onto nitrocellulose membrane using Bio-Rad Trans Blot apparatus. Membranes were washed with $20 \mathrm{mM}$ Tris HCL, pH 7.5, containing $0.1 \%$ Tween 20 and $150 \mathrm{mM} \mathrm{NaCl}$ (Tris-buffered saline, TBST) and blocked with TBST containing $5 \%$ nonfat dry milk for 2 hours at room temperature. Next, membranes were incubated with primary antibody overnight at $4^{\circ} \mathrm{C}$ in $3 \%$ bovine serum albumin in TBST and washed with TBST, followed by incubation with corresponding secondary antibody conjugated with horseradish peroxidase for 1 hour at room temperature. Immunoblots were visualized using chemiluminescent reagent (Pierce Chemicals, Rockford, IL). An LAS-3000 imaging system (Fujifilm Life Sciences, Stamford, CT) was used to capture chemiluminescence, and Multi Gauge software (Fujifilm Life Sciences) was used for quantitation of immunoblots. The chemiluminescence intensity was within the linear range of the detection. $\beta$-Actin and/or histone $\mathrm{H} 1$ protein was used for equalizing protein loading. Digitalized data were normalized to controls and statistically analyzed using GraphPad Prism 4 (GraphPad Software, San Diego, CA).

Histology. Formalin-fixed liver tissue was embedded in paraffin, sectioned, and stained with H\&E) at the University of Missouri Research Animal Diagnostic Laboratory, Columbia, MO, as described earlier (Aroor et al., 2011). Microscopic images of slides were taken using a Nikon Eclipse E600 (Nikon, Inc., Tokyo, Japan) with Nikon Digital Camera, DXM 1200 and Nikon ACT-1 v2.70.

Lipid Vesicle Determinations. For a semiquantitative determination of the vesicle numbers in the images, a section of histology images was selected and divided into four quadrants; vesicles were counted and recorded. Data are from four male control, four male ethanol, four female control, and four female ethanol samples. Data from each group were pooled together for calculations.

Hepatic Triglyceride Assay. Liver triglyceride levels were determined essentially as reported earlier (Aroor et al., 2011). Liver tissue $(30 \mathrm{mg}$ ) was homogenized in $0.5-\mathrm{ml}$ hypotonic buffer containing $20 \mathrm{mM}$ Tris, $2 \%$ Triton X-100, and Sigma protease inhibitor cocktail (p8340) in a 2-ml- capacity Dounce homogenizer with pestle A. The sample was heated to $60^{\circ} \mathrm{C}$, followed by centrifugation at $13,000 \mathrm{~g}$ for 5 minutes. The supernatant was used for triglyceride estimation using the assay kit and protocol provided by the supplier (cat. no. F6428; Sigma-Aldrich Company, St. Louis, MO). Results are reported as milligrams per gram of liver tissue. 
Analysis of Phosphatidylethanol. The frozen liver tissues from rats were homogenized in saline on ice. The homogenates were then subjected to a modified Bligh-Dyer extraction (Bligh and Dyer, 1959) with the addition of internal standard 14:0/14:0 (sn-1/sn-2 aliphatic groups indicated as the number of carbons per number of double bonds) phosphatidylethanol (Peth). PEth internal standard was synthesized using phospholipase D as previously described (Omodeo et al.,1989), yielding $>90 \%$ conversion of 14:0/14:0 PC (Avanti Polar Lipids, Alabaster, AL) to 14:0/14:0 PEth as determined by electrospray ionization/mass spectrometry (ESI/MS). Unreacted 14:0/14: 0 PC (Avanti Polar Lipids) and phosphatidic acid were separated from product, 14:0/14:0 PEth $(\mathrm{Rf}=0.56)$, using thin-layer chromatography with chloroform/methanol/water (16/6/1, v/v/v), and purity was confirmed to be $>95 \%$ by ESI/MS. Subsequently, 14:0/14:0 PEth was quantified by ESI-MS with comparisons to known amounts of commercially available 16:0/18:1 PEth (Avanti Polar Lipids), and quantification was confirmed using gas chromatography-flame ionization detector of the fatty acid methyl ester derivatives of 14:0/14:0 PEth (e.g., tetradecanoyl methyl ester) compared with the derivative of the internal standard heptadecanoic acid (Gross,1984).

The lipid extracts were stored under nitrogen in chloroform until they were dried under nitrogen and suspended in methanol/chloroform $(4 / 1, v / v)$ to be quantified by ESI-MS/MS on a triple-quadrupole instrument (Thermo Fisher Quantum Ultra) using shotgun lipidomics methods (Han and Gross, 2005). PEth species were quantified in negative-ion mode. PEth molecular species were detected by product ion scanning of $\mathrm{m} / z 181.2$, and aliphatic group assignments were confirmed by product ion scanning of fatty acids associated with phospholipid species. Individual molecular species were quantified by comparing their ion intensities with the respective lipid class internal standards, with the addition of type I and II $\left[{ }^{13} \mathrm{C}\right]$ isotope corrections (Han and Gross, 2005; Rong et al., 2017).

Statistical Analysis. GraphPad Prism 4 was used for calculations. Data were analyzed by analysis of variance and $P$ values less than $0.05(P<0.05)$ were considered statistically significant.

\section{Results}

In this in vivo study, we compared the effects of acute binge alcohol on histopathological and biochemical changes in male and female rat livers. A diverse set of parameters were examined, including blood alcohol concentration (BAC), histology, pathologic markers (ALT, Cyp2E1, catalase, caspase 3), steatosis (fat vesicles, triglyceride), MAPK signaling components (ERK1/2, p38 MAPK, JNK), transcription related proteins (CREB, PGC1 $\alpha$, c-fos, c-myc), and epigenetic markers (site-specific histone modifications). We also determined the levels of PEth, a phospholipid exclusively generated after ethanol exposure, and the protein levels of DGK $\alpha$. The goal was to establish the differences in treatment of binge alcohol treatment of males and females using diverse set of parameters of liver function and pathology.

Our groups of four rats provided statistically significant differences in many parameters between the two sexes. Data showed low variability and were consistent and reliable, which can be considered as the robustness of the responses in females that are distinct from males. It is possible that additional differences may be found if more animals are used, but we are not suggesting that other parameters where we have not observed statistically significant differences are "sexindependent." The details of the data are described below.

Histologic and Pathologic Changes. Three doses of acute binge alcohol $(5 \mathrm{~g} / \mathrm{kg})$ by the intragastric route, 12 hours apart, caused both histologic and pathologic alterations in the livers of the two sexes but with dramatic differences. The BAC levels in females were about twice that measured in males (Fig. 1A). For calculation of the fold changes, we used sex matches (i.e., male control for male ethanol, female control for female ethanol throughout this study). Data are presented as fold change over control of the respective sex. The histologic changes were also more pronounced in samples from females than in samples from males (Fig. 1B). For example, although both sexes showed binge alcohol-induced fatty vesicle increase, the liver of females contained larger fatty vesicles (macrosteatosis). Females had about four times more vesicles than did the males (Fig. 1C). The liver triglyceride values were also consistent with this pattern (Fig. 1D) wherein triglyceride values were increased by about 2 -fold in males and 5 -fold in females compared with their controls. The ALT levels, a marker of necrosis, were relatively unchanged in males but increased about $50 \%$ over controls in females (Fig. 1E). In contrast, the apoptotic marker caspase 3 levels were decreased by binge alcohol in both males and females. Their levels in livers from females were slightly higher than in those of males after binge ethanol (Fig. 1F). Binge alcohol administration might favor necrotic injury in lieu of apoptotic changes, but female rat livers could still be more susceptible to apoptotic damage than male livers.

Of other pathologic markers we measured, CYP2E1 protein levels were increased by binge alcohol in both males (2-fold) and females (2.5-fold), with higher levels in females (Fig. 2A). Compared with controls, catalase protein levels appeared to decrease in livers of males after binge but were unaltered in females' livers (Fig. 2B).

Changes in MAPK Signaling Components by Binge Alcohol. Acute and chronic alcohol causes alterations in protein kinases in the liver. We examined the levels of various MAP kinases, their phosphorylated levels, and the ratio of phospho-to-protein levels in the two sexes. After binge ethanol administration, the levels of ERK protein were increased to similar extents in livers of males and females, but the phospho-ERK, although increased in both sexes, was much less robust in females compared with males (Fig. 3A), reflected in a decrease in the ratio of P-ERK/ERK in ethanol-treated females compared with males. The protein levels of p38 MAPK were not changed in males or females after binge alcohol (Fig. 3B); however, the phospho-p38 levels increased in both males and females, more in males. This finding agreed with the phospho-p38/p38 protein ratio (Fig. 3B). In the case of JNK protein, in females, there was an insignificant increase (Fig. 3C), but phospho levels were similar in both sexes. There was a concomitant decrease in the ratio of P-JNK/JNK (Fig. 3C).

Changes in Transcription-Related Proteins. Compared with control values, the CREB protein level was elevated by about $75 \%$ in females, but it was unchanged in males (Fig. 4A). The increase in phospho-CREB, surprisingly, was greater in males than females after binge (Fig. 4B). Interestingly, the ratio of phospho-CREB/CREB increased about 2-fold over controls in males but decreased by $20 \%$ in females compared with controls (Fig. 4C). The levels of PGC1 $\alpha$ protein were unaffected by binge alcohol in livers of males but were elevated in female livers by about $30 \%$ (Fig. 4D). The c-fos protein levels after binge alcohol were elevated by $50 \%$ over controls in males but were reduced substantially to about $20 \%$ of the control in livers of females (Fig. 4E). The levels of c-MYC protein did not change much by acute binge in either sex (Fig. 4F). 
A

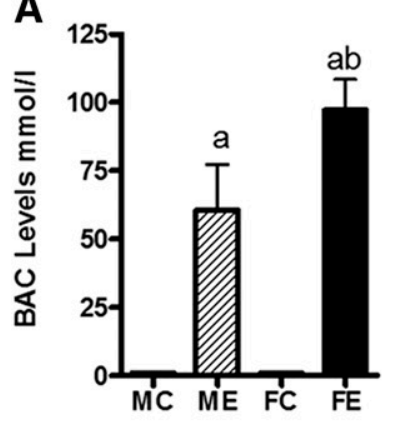

B

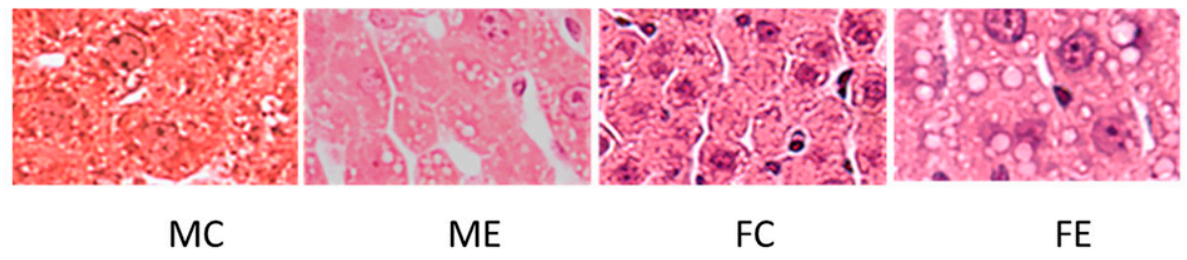

C

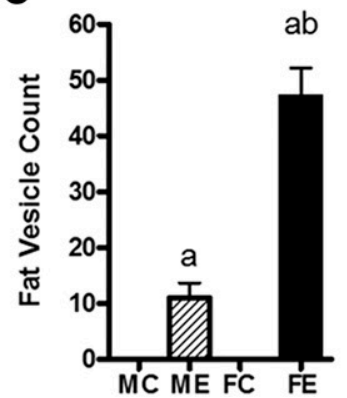

D

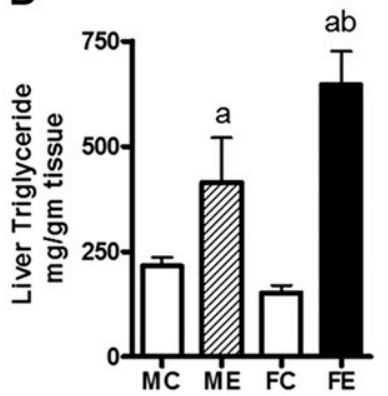

E

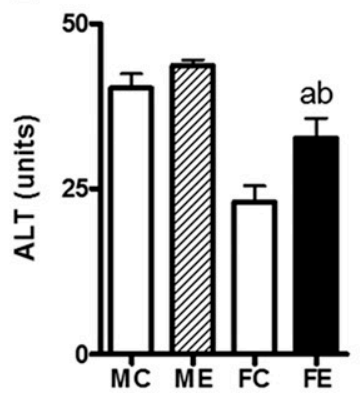

F

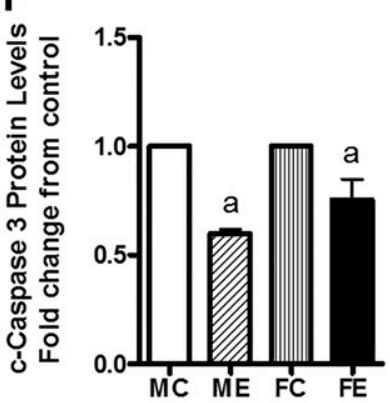

Fig. 1. Histologic and pathologic changes in ethanol binge treated rats. Ethanol three-repeat binge treatments (each $5 \mathrm{~g} / \mathrm{kg}, 12 \mathrm{hours}$ apart) in male and female rats (groups of four) were performed as described under Materials and Methods. Four hours after the last binge, blood samples and liver tissues were collected. (A) Blood ethanol concentration. (B) Histology image showing differences in lipid vesicles. (C) Count of lipid vesicles. (D) Triglyceride levels. (E) Serum ALT levels. (F) Cleaved caspase-3. Values are mean \pm S.E.M. ( $n=4$ rats). For (A and C-E), the values are shown on $y$-axis. For (F), values are presented as fold increase over respective sex controls. FC, female control; FE, female ethanol binge; MC, male control; ME, male ethanol binge. a,Significant compared with control $(P<0.05)$; b, significant compared with the male ethanol binge group; $(P<0.05)$.

Binge Alcohol-Induced Changes in Epigenetic Markers. We next investigated the binge ethanol-induced changes in the site-specific histone modifications. Levels of acetylated H3AcK9 increased in both male and female rats but without any difference between the two sexes (Fig. 5A). A similar profile was seen with dually modified phosphoacetylated histone H3 (i.e., H3AcK9PS10; Fig. 5B). On the other hand, the levels of H3PS10 and H3PS28 were not altered by binge alcohol in males or females (Fig. 5, C and D).

Binge Alcohol-Induced Changes in PEth and DGK $\boldsymbol{\alpha}$. Phosphatidylethanol (PEth) is a phospholipid that is exclusively produced in the presence of ethanol (Aroor et al., 2002; Aradottir et al., 2006; Viel et al., 2012). We examined the profile of various PEth species using ESI-MS/MS on a triplequadruple instrument using shotgun lipidomics methodology. The total basal amount of PEth is about 2-fold greater in the livers of females compared with those in males. The small basal PEth level is intriguing and may arise from endogenous metabolic reactions, including that in gut; however, the increase in PEth after binge ethanol was 10-fold greater than controls in males versus 5 -fold in females. The total quantity of PEth after binge ethanol did not different much in livers of males compared with those of females (Fig. 6). When individual PEth species were evaluated, differences were seen. PEth species 34:2, 36:4, and 38:5 were reduced in females compared with males after binge ethanol treatment. On the other hand, the species 36:2 was elevated in females compared with males. Other species $(34: 1,36: 3,36: 5,38: 6$, and 38:4) showed no differences (Fig. 6).

Diacylglycerol is produced in cells from various lipid turnover and remodelling pathways, including that via triglyceride lipase, phosphatidate phosphohydrolase, and phospholipase C (Van der Veen et al., 2017). Diacylglycerol kinase- $\alpha(\mathrm{DGK} \alpha)$ is a lipid-metabolizing enzyme that converts DG to phosphatidic acid (PA), which can be further converted to lyso-PA and other metabolites. DGK $\alpha$ is proposed to have role(s) in neoplasm and in the promotion of cancer (see Purow, 2015), and its gene levels were recently shown to be elevated in the brains of mice after binge alcohol (Finn et al., 2018). We therefore determined the protein levels of DGK $\alpha$ and found that, in females, there was a large increase, about $95 \%$, in this
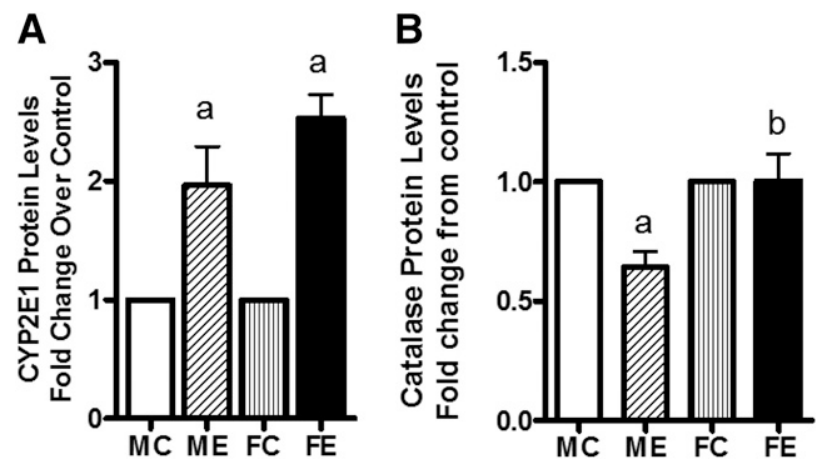

Fig. 2. Levels of CYP2E1 (A) and catalase (B). After binge treatments (as in Fig. 1), the whole-cell extracts were prepared (see Materials and Methods). The protein levels of these components were determined by Western blotting. Fold change over respective male or female control values are presented as mean \pm S.E.M. ( $n=4$ rats $)$. a, Significant compared with control $(P<0.05)$; b, significant compared with the male ethanol binge group; $(P<0.05)$. FC, female control; FE, female ethanol binge; MC, male control; ME, male ethanol binge. 
A

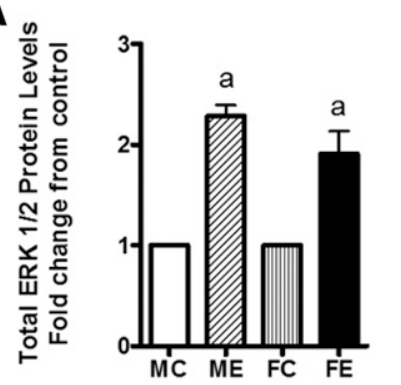

B

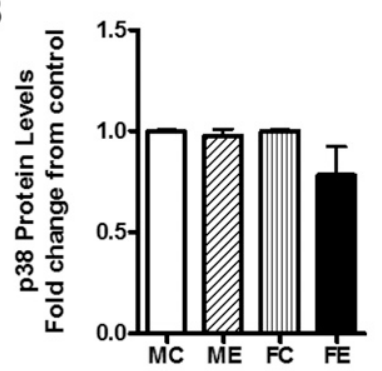

C

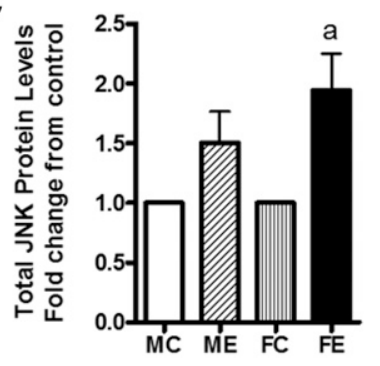

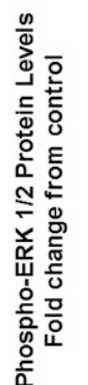
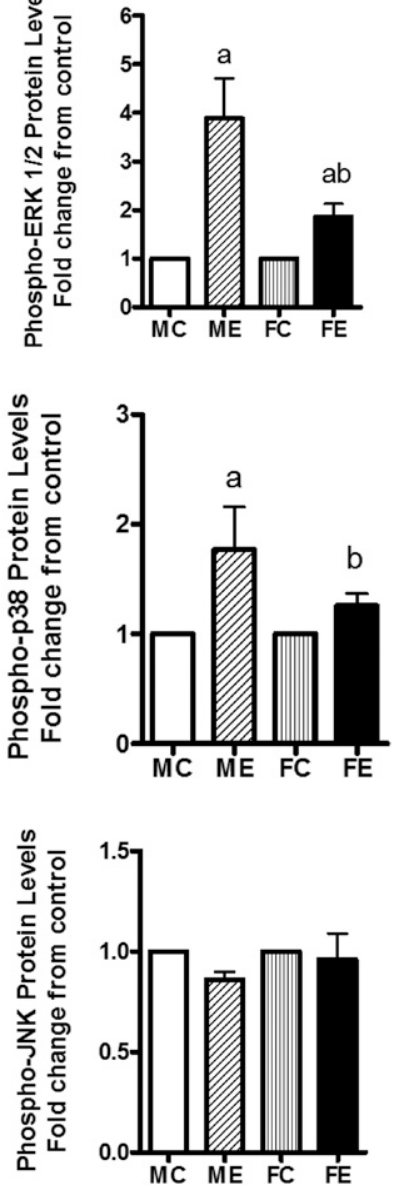

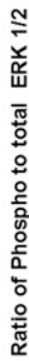

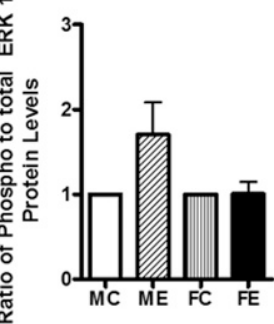

Fig. 3. Changes in MAPK signaling components. Levels of total ERK 1/2, phospho-ERK $1 / 2$, and ratio of phospho- to total ERK 1/2 (A); levels of total p38, phospho-p38, and ratio of phospho- to total p38 (B); and levels of Total JNK, phospho-JNK, and ratio of phospho- to total JNK (C) are presented. The experimental details were as described in Fig. 2. The protein levels of these components in wholecell extracts were determined by Western blotting. As in Fig. 2, the values are mean \pm S.E.M. ( $n=4$ rats). a, Significant compared with control $(P<0.05)$; b, significant compared with the male ethanol binge group; $(P<0.05)$. FC, female control; FE, female ethanol binge; $\mathrm{MC}$, male control; $\mathrm{ME}$, male ethanol binge.

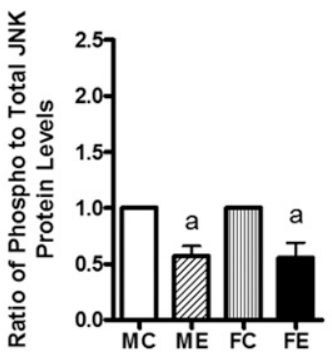

protein compared with only about $20 \%$ in males after binge alcohol treatment (Fig. 7).

\section{Discussion}

This report is the first to demonstrate differences in the pattern and profile of the commonly known indices of liver physiology and pathology between male and female rats exposed to three repeat acute binge alcohol treatments in vivo. A diverse set of parameters were examined related to the pathophysiology of liver, epigenetics, and cell signaling responses. In brief, three categories of data were observed: 1) data that showed significant differences in males and females, 2) data that showed alterations by binge ethanol levels but with negligible differences in males and females, and 3) data showing no alterations in response to ethanol and no difference between the sexes (Table 1).

Liver injury, characterized by hepatic steatosis, was seen in both male and female rats, but the magnitude of steatosis was greater in female rats. This increase in steatosis was also associated with increased serum transaminase, suggesting more hepatic injury in females. The caspase-3 levels were decreased in both male and female mice, suggesting differential regulation of necrosis and apoptosis after binge. Binge alcohol increased blood alcohol levels in both male and female rats, but levels in females were significantly higher. Body weight, fat, and age can influence alcohol metabolism.
Variations in these levels can result in different levels of BAC. Since male and female rats used here were of the same age and weight and were administered the same amount of alcohol, it can be ruled out that differences in BAC are due to differences in body size and suggests that differences in BAC are due to sex. It has been reported that gastric alcohol dehydrogenase activity and first-pass metabolism are decreased in women, resulting in higher blood alcohol levels (Frezza et al., 1990). As shown by our results, although a greater alcohol level was seen in animals with more steatosis, other markers of injury or signaling pathways that are known to be modulated by alcohol were variably affected in males versus females, thereby supporting the conclusion that BAC is not solely responsible for all the changes observed. Increased alcohol concentration in blood may contribute to increased liver injury, although others have suggested that liver injury may be related to altered gut microbiome and independent of blood alcohol levels (Kirpich and McClain, 2017). Enhanced ethanol metabolism and associated oxidative stress have also been considered as factors contributing to hepatic injury (Shukla and Aroor, 2006; Shukla et al., 2013a). An increase in CYP2E1 in females compared with males may contribute to increased injury in females. With higher levels of ethanol in females, it is likely that higher levels of acetaldehyde and acetate will be present. This likelihood, together with the roles of CYP2E1 and catalase in the increased susceptibility of females to alcohol, remains to be studied. 
A

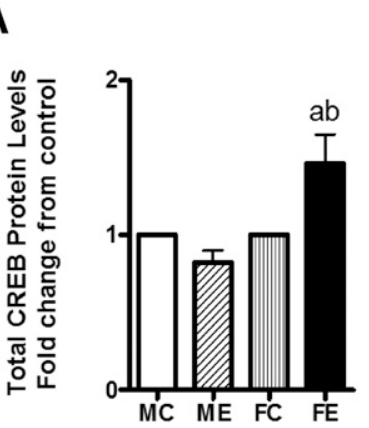

B

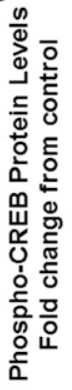

C

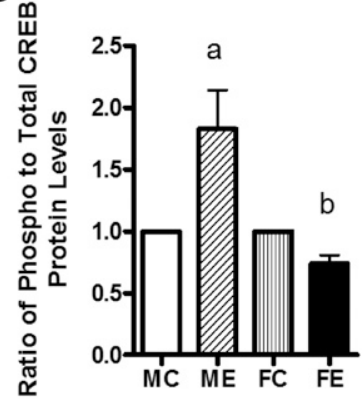

D

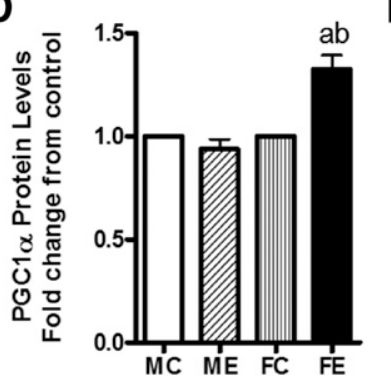

$E$

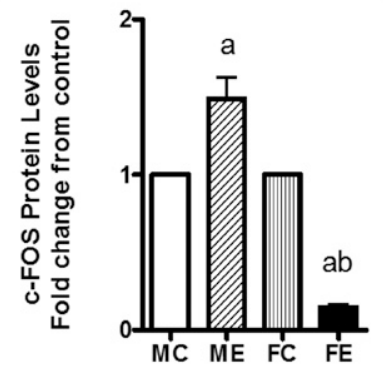

$\mathbf{F}$

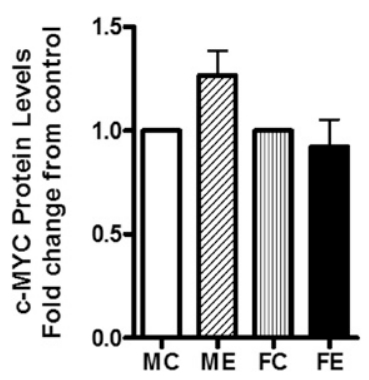

Fig. 4. Changes in transcription related factors. Protein levels of total CREB (A); phosphoCREB (B); ratio of phospho- to total CREB (C); PGC-1 $\alpha$ (D); c-FOS (E); and c-MYC (F) are shown. The protein levels of these components were determined by Western blotting. As in Fig. 2, values are fold change over respective sex controls and are mean \pm S.E.M. $(n=4$ rats). a, Significant compared with control $(P<0.05)$; b, significant compared with the male ethanol binge group; $(P<0.05)$. FC, female control; $\mathrm{FE}$, female ethanol binge; $\mathrm{MC}$, male control; ME, male ethanol binge.
Changes in mitogen-activated protein kinases (MAPKs) comprising extracellular regulated kinases 1/2 (ERK1/2), c-Jun N-terminal kinase (JNK), and p38 MAPK related to alcoholic liver injury after acute, chronic, and binge alcohol administration have been shown earlier in male rats (Aroor and Shukla, 2004; Lee and Shukla, 2005; Venugopal et al., 2007; Apte et al., 2007; Mandrekar and Szabo, 2009). It has also been proposed that the acute effects of ethanol may be related to the prolongation of ERK1/2 activation (Nguyen and Gao, 2002; Hsu et al., 2006); however, sex differences in the pattern of activation of all three MAPKs during alcohol binge administration have not been examined until now. In this study, the notable finding in relation to MAP kinase signaling is the highly significant increase in ERK1/2 and p38 MAPK phosphorylation in male rats after alcohol binge compared with a more moderate increase in phosphorylation of ERK1/2 and p38MPAK in female rats. A role of ERK1/2 activation in hepatic steatosis and necrosis is gradually being recognized (Nguyen and Gao, 2002; Kaizu et al., 2008). Administration of MEK inhibitor U-0126 was shown to suppress endotoxin-induced liver injury after sensitization induced by acute administration of ethanol in mice (Beier et al., 2009), and ERK1/2 activation has been shown to mediate arachidonic acid-induced hepatocyte necrosis in oxidatively stressed CYP2E1-overexpressing rat hepatocytes (Schattenberg et al., 2004). Our previous proteome studies on acute alcohol binge administration in male rats showed increased expression of CYP2E1, together with microvascular steatosis (Aroor et al., 2011). It is therefore somewhat surprising to see that the magnitude of ERK1/2 phosphorylation was greater in male rats compared with female rats, but steatosis was more marked in female rats. This finding may be reconciled by noting that enhanced phosphorylation of p38 MAPK and ERK1/2 in male rats coincided with a concomitant larger increase in phosphorylation of CREB in male rats. In this regard, CREB is known to be phosphorylated by both p38 MAPK and ERK1/2 and has been implicated in the regulation of hepatic triglyceride accumulation (Aroor et al., 2012a). Impairment of CREB signaling using dominant negative CREB in vivo promotes
A

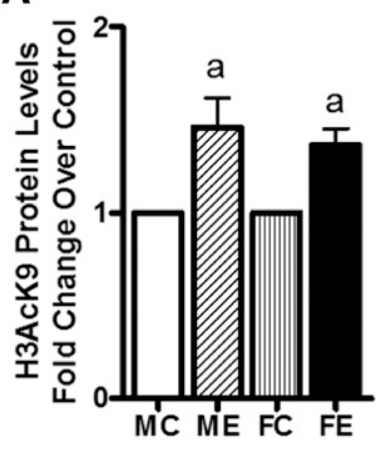

C

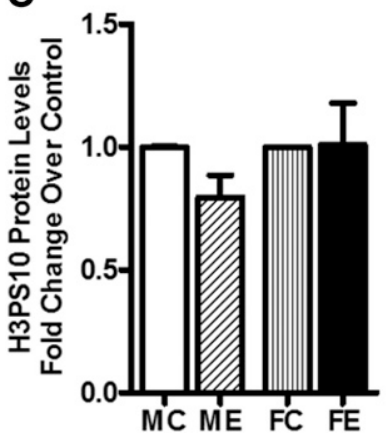

B

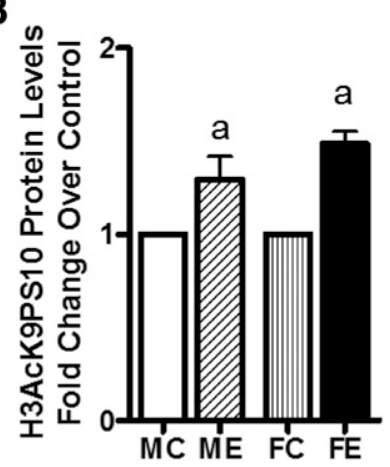

D

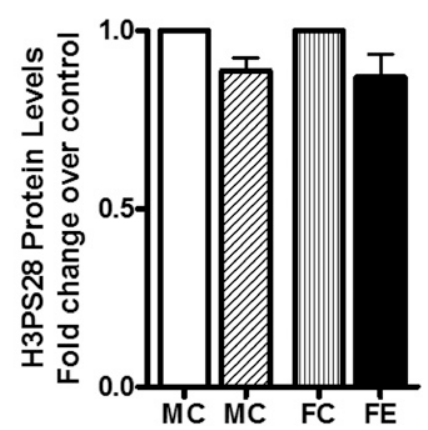

Fig. 5. Histone $\mathrm{H} 3$ acetylation and phosphorylations. Levels of H3AcK9 (A); H3AcK9PS10 (B); H3PS10 (C); and H3PS28 (D) are presented. The experimental details were as described in Fig. 2. The levels of these components were determined by Western blotting. Values are fold change over respective sex control and are mean \pm S.E.M. $(n=4$ rats $)$. a, Significant compared with control $(P<0.05)$; b, significant compared with the male ethanol binge group; $(P<0.05)$. FC, female control; $\mathrm{FE}$, female ethanol binge; $\mathrm{MC}$, male control; $\mathrm{ME}$, male ethanol binge. 

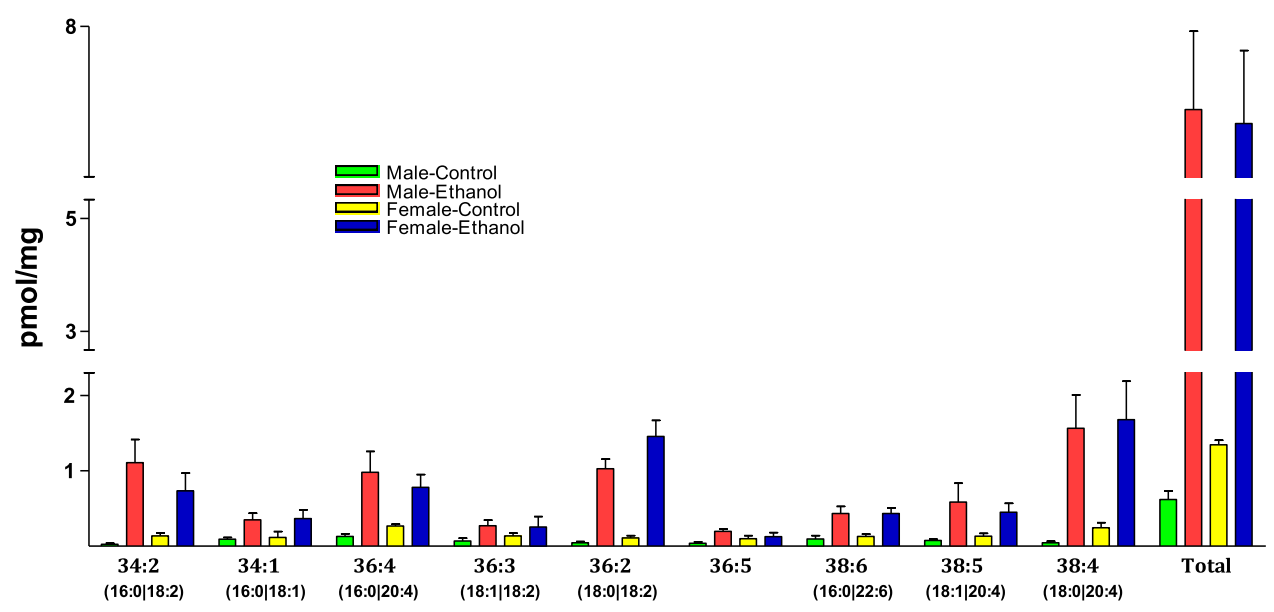

Phosphatidylethanol (PEth) Species
Fig. 6. Analysis of PEth species. Lipids were extracted from liver samples and PEth analyzed by ESI-MS/MS on a triplequadrupole instrument using shotgun lipidomics method (see Materials and Methods). Values (mean \pm S.D.) are from four male or female rats and are presented as picomoles per milligram of tissue. development of steatosis (Herzig et al., 2003; Puigserver and Spiegelman, 2003; Shukla et al., 2015a). Therefore, it is likely that impairment of CREB phosphorylation in female rats may interfere with CREB signaling and contribute in part to the development of increased hepatic steatosis after alcohol binge in female rats. In contrast to our earlier in vitro studies with hepatocytes (Lee and Shukla, 2005), we have not observed increased JNK phosphorylation in this study. Liver regeneration subsequent to hepatectomy or chemical injury is associated with differential regulation of c-myc and c-fos (Ohri et al., 2002); however, c-fos and c-myc were not affected during liver regeneration after chronic ethanol injury (Diehl et al., 1990). In the present study, female rat liver c-fos protein levels were significantly reduced after alcohol binge, which may suggest a role for c-fos in the susceptibility of the liver to binge alcohol injury in females, leading to impaired reparative responses.

Epigenetic histone modifications caused by acute, chronic, and acute on chronic binge alcohol administration results in alterations in the expression of gene related to hepatic steatosis, alcohol metabolism, and inflammatory response in a gene-selective manner (see Shukla and Zakhari, 2013b); however, sex differences in epigenetic histone modifications have not been investigated. We examined site-specific modifications in histone H3. Although histone serine phosphorylation was not changed after alcohol binge, histone H3K9 acetylation and H3K9PS10 phosphoacetylation increased to a similar magnitude after alcohol binge in both male and female rats. Previously we have reported role of p38 MAPK and ERK1/2 in the regulation of histone phosphorylation and acetylation (James et al., 2012). Absence of histone phosphorylation, despite changes in p38MAPK, and similar increases in acetylation and phosphoacetylation of histone H3 in males and females, despite differential activation of ERK1/2, suggest additional mechanisms that may be MAPK independent. For example, we have reported that histone $\mathrm{H} 3$ acetylation can be affected by oxidative stress and acetate accumulation (Park et al., 2005; Choudhury et al., 2010, 2011; Shukla et al., 2015b). Increased histone H3 acetylation was considered associated with gene-specific upregulation of fatty acid synthase gene and downregulation of carnitine palmitoyl transferase 1a gene, thereby contributing to increased synthesis of triglyceride and impaired oxidation of fatty acid (Kirpich et al., 2013).
This report is the first to describe the intriguing pattern and differences in individual PEth species formed in the livers of male and female rats exposed to binge alcohol. Although total PEth levels after binge ethanol were rather similar in the two sexes (male erhanol vs. female erhanol), the levels of PEth species 36:2 increased in females, and others decreased (e.g., 34:2, 36:4, 36:5) or did not change (e.g., 34:1, 36:3, 38:6, 38:4). Such approaches, therefore, have the potential to identify and examine the specific roles of individual PEth species uniquely generated after alcohol exposure. It is noteworthy in the present study that DGK $\alpha$ levels increased much more in females than in males after binge ethanol. DGK $\alpha$ is known to be involved in the promotion of neoplastic growth (Dominguez et al., 2013), triglyceride metabolism (Van der Veen et al., 2017), T-cell receptor signaling/immunologic responses (Purow, 2015), and development of fibrosis (Weigel et al., 2016). Whether females are at increased risk for the development of cancer with binge ethanol needs to be further

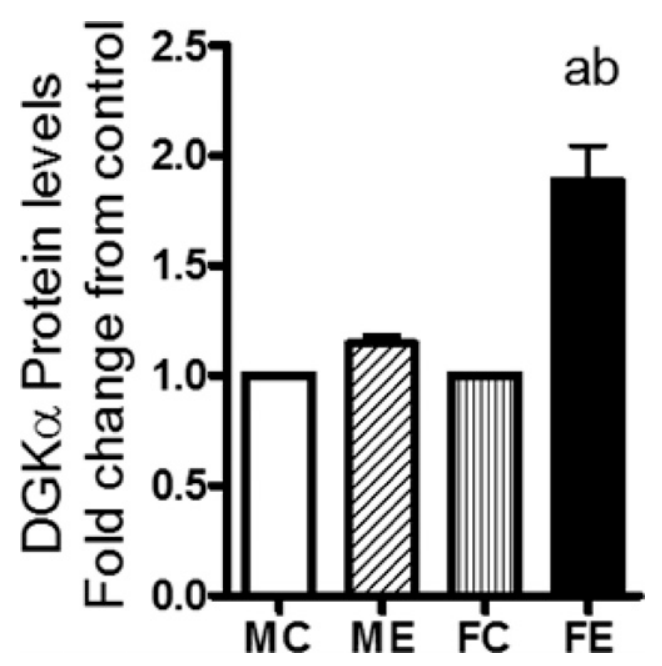

Fig. 7. DGK $\alpha$ protein levels. In whole-cell extracts, DGK $\alpha$ protein levels in WCE were determined by Western blotting, followed by densitometric quantification. Increases in the protein levels after binge ethanol are presented as fold increases over respective sex controls. The experimental details were as described in Fig. 2 . Values are mean \pm S.E.M. ( $n=4$ rats). a, Significant compared with control $(P<0.05)$; b, significant compared with the male ethanol binge group; $(P<0.05)$. FC, female control; FE, female ethanol binge; MC, male control; ME, male ethanol binge. 
TABLE 1

Summary of the three categories of data

\begin{tabular}{cl}
\hline \multicolumn{1}{c}{ Category } & \multicolumn{1}{c}{ Parameter } \\
\hline $\begin{array}{c}\text { Show significant differences } \\
\text { in sexes }\end{array}$ & Histology \\
& BAC, ALT, TG, lipid vesicles \\
& Catalase \\
& TOTAL CREB, phospho-CREB, \\
& c-fos, PGC1 $\alpha$, \\
& Phospho-ERK 1/2, phospho-p38 \\
& Diacylglycerol kinase $\alpha$ \\
Histone H3AcK9, H3AcK9PS10 \\
CYPow change but negligible & Total ERK1/2, total JNK \\
differences in sexes & Phosphatidylethanol \\
& Histone H3SP10, H3SP28 \\
c-MYC & Phospho-JNK, total p38 MAPK \\
differences in sexes &
\end{tabular}

ALT, alanine aminotransferase; BAC, blood alcohol concentration; CREB, cyclic AMP response element binding protein; CYP2E1, cytochrome P4502E1; ERK, extracellular signal-regulated kinase; H3AcK9, histone H3 acetylated at Lys 9; H3PS10, histone H3 phosphorylated at Ser10; H3PS28, histone H3 phosphorylated at ser28; JNK, C-jun N-terminal kinase; MAPK, mitogen-activated protein kinase; PGC1 $\alpha$, peroxisome proliferator-activated receptor $\gamma$-coactivator $1 \alpha ; \mathrm{TG}$, triglyceride.

investigated, particularly in breast cancer, where a strong relationship with alcohol use is known (Shield et al., 2016).

Based on this study, it is suggested that acute binge ethanol effects are not "all or none" responses in male and female. The fact that some indices changed but others did not change in males versus females is reflective of selective susceptibilities. The greater accumulation of triglycerides and enhanced liver injury in female rats in conjunction with dysregulated MAPK signaling, oxidative stress related to alcohol metabolism, and specific alterations in PEth and DGK $\alpha$ may be involved in binge-induced increased injury in females, setting the stage for additional studies to decipher the sex differences associated with binge alcohol. In conclusion, acute binge ethanol in vivo selectively modifies molecular pathways in the livers of males and females, leading to increased susceptibilities in females.

\section{Authorship Contributions}

Participated in research design: Shukla, Aroor.

Conducted experiments: Shukla, Aroor, Restrepo, Liu, Franke.

Performed data analysis: Shukla, Aroor, Restrepo, Lim, Franke, Ford, Korthuis.

Wrote or contributed to the writing of the manuscript: Shukla, Aroor, Restrepo, Lim, Ford, Korthuis.

\section{References}

Abdelmegeed MA, Banerjee A, Jang S, Yoo SH, Yun JW, Gonzalez FJ, Keshavarzian A, and Song BJ (2013) CYP2E1 potentiates binge alcohol-induced gut leakiness, steatohepatitis, and apoptosis. Free Radic Biol Med 65:1238-1245.

Apte M, McCarroll J, Pirola R, and Wilson J (2007) Pancreatic MAP kinase pathways and acetaldehyde. Novartis Found Symp 285:200-216.

Aradottir S, Asanovska G, Gjerss S, Hansson P, and Alling C (2006) PHosphatidylethanol (PEth) concentrations in blood are correlated to reported alcohol intake in alcohol-dependent patients. Alcohol Alcohol 41:431-437.

Aroor AR, Custer GW, Weng YI, Lee YJ, and Shukla SD (2002) Phosphotidylethanol mimics ethanol modulation of $\mathrm{p} 42 / 44$ mitogen-activated protein kinase signalling in hepatocytes. Alcohol Alcohol 37:534-539.

Aroor AR, Jackson DE, and Shukla SD (2011) Elevated activation of ERK1 and ERK2 accompany enhanced liver injury following alcohol binge in chronically ethanol-fed rats. Alcohol Clin Exp Res 35:2128-2138.

Aroor AR, Jackson DE, and Shukla SD (2012a) Dysregulated phosphorylation and nuclear translocation of cyclic AMP response element binding protein (CREB) in rat liver after chronic ethanol binge. Eur J Pharmacol 679:101-108.

Aroor AR, James TT, Jackson DE, and Shukla SD (2010) Differential changes in MAP kinases, histone modifications, and liver injury in rats acutely treated with ethanol. Alcohol Clin Exp Res 34:1543-1551.
Aroor AR, Restrepo RJ, Kharbanda KK, and Shukla SD (2014) Epigenetic histone modifications in a clinically relevant rat model of chronic ethanol-binge-mediated liver injury. Hepatol Int 8 (Suppl 2):421-430.

Aroor AR, Roy LJ, Restrepo RJ, Mooney BP, and Shukla SD (2012b) A proteomic analysis of liver after ethanol binge in chronically ethanol treated rats. Proteome Sci 10:1-12.

Aroor AR and Shukla SD (2004) MAP kinase signaling in diverse effects of ethanol. Life Sci 74:2339-2364.

Axley PD, Richardson CT, and Singal AK (2019) Epidemiology of alcohol consumption and societal burden of alcoholism and alcoholic liver disease. Clin Liver Dis 23 : 39-50.

Bardag-Gorce F, Oliva J, Wong W, Fong S, Li J, French BA, and French SW (2010) S-adenosylmethionine decreases the peak blood alcohol levels $3 \mathrm{~h}$ after an acute bolus of ethanol by inducing alcohol metabolizing enzymes in the liver. Exp Mol Pathol 89:217-221.

Becker U, Deis A, Sørensen TI, Grønbaek M, Borch-Johnsen K, Müller CF, Schnohr $\mathrm{P}$, and Jensen G (1996) Prediction of risk of liver disease by alcohol intake, sex, and age: a prospective population study. Hepatology 23:1025-1029.

Beier JI, Luyendyk JP, Guo L, von Montfort C, Staunton DE, and Arteel GE (2009) Fibrin accumulation plays a critical role in the sensitization to lipopolysaccharideinduced liver injury caused by ethanol in mice. Hepatology 49:1545-1553.

Bligh EG and Dyer WJ (1959) A rapid method of total lipid extraction and purification. Can J Biochem Physiol 37:911-917.

Breslow RA, Castle IP, Chen CM, and Graubard BI (2017) Trends in alcohol consumption among older Americans: National Health Interview Surveys, 1997 to 2014. Alcohol Clin Exp Res 41:976-986.

Choudhury M, Pandey RS, Clemens DL, Davis JW, Lim RW, and Shukla SD (2011) Knock down of GCN5 histone acetyltransferase by siRNA decreases ethanolinduced histone acetylation and affects differential expression of genes in human hepatoma cells. Alcohol 45:311-324.

Choudhury M, Park PH, Jackson D, and Shukla SD (2010) Evidence for the role of oxidative stress in the acetylation of histone H3 by ethanol in rat hepatocytes. Alcohol 44:531-540.

Diehl AM, Thorgeirsson SS, and Steer CJ (1990) Ethanol inhibits liver regeneration in rats without reducing transcripts of key protooncogenes. Gastroenterology 99: 1105-1112.

Dominguez CL, Floyd DH, Xiao A, Mullins GR, Kefas BA, Xin W, Yacur MN Abounder R, Lee JK, Wilson GM, et al. (2013) Diacylglycerol kinase $\alpha$ is a critical signaling node and novel therapeutic target in glioblastoma and other cancers. Cancer Discov 3:782-797.

Fernandes LMP, Cartágenes SC, Barros MA, Carvalheiro TCVS, Castro NCF, Schamne MG, Lima RR, Prediger RD, Monteiro MC, Fontes-Júnior EA, et al (2018) Repeated cycles of binge-like ethanol exposure induce immediate and delayed neurobehavioral changes and hippocampal dysfunction in adolescent female rats. Behav Brain Res 350:99-108.

Finn DA, Hashimoto JG, Cozzoli DK, Helms ML, Nipper MA, Kaufman MN, Wiren $\mathrm{KM}$, and Guizzetti M (2018) Binge ethanol drinking produces sexually divergent and distinct changes in nucleus accumbens signaling cascades and pathways in adult C57BL/6J mice. Front Genet 9:325.

Frezza M, di Padova C, Pozzato G, Terpin M, Baraona E, and Lieber CS (1990) High blood alcohol levels in women. The role of decreased gastric alcohol dehydrogenase activity and first-pass metabolism. N Engl J Med 322:95-99.

Fulham MA and Mandrekar P (2016) Sexual dimorphism in alcohol induced adipose inflammation relates to liver injury. PLoS One 11:e0164225.

Ghosh Dastidar S, Warner JB, Warner DR, McClain CJ, and Kirpich IA (2018) Rodent models of alcoholic liver disease: role of binge ethanol administration. Biomolecules 8 (1):1-21.

Gross RW (1984) High plasmalogen and arachidonic acid content of canine myocardial sarcolemma: a fast atom bombardment mass spectroscopic and gas chromatography-mass spectroscopic characterization. Biochemistry 23:158-165.

Han X and Gross RW (2005) Shotgun lipidomics: electrospray ionization mass spectrometric analysis and quantitation of cellular lipidomes directly from crude extracts of biological samples. Mass Spectrom Rev 24:367-412.

Herzig S, Hedrick S, Morantte I, Koo SH, Galimi F, and Montminy M (2003) CREB controls hepatic lipid metabolism through nuclear hormone receptor PPARgamma. Nature 426:190-193.

Hsu MK, Qiao L, Ho V, Zhang BH, Zhang H, Teoh N, Dent P, and Farrell GC (2006) Ethanol reduces p38 kinase activation and cyclin D1 protein expression after partial hepatectomy in rats. J Hepatol 44:375-382.

Iimuro Y, Frankenberg MV, Arteel GE, Bradford BU, Wall CA, and Thurman RG (1997) Female rats exhibit greater susceptibility to early alcohol-induced liver injury than males. Am J Physiol 272:G1186-G1194.

James TT, Aroor AR, Lim RW, and Shukla SD (2012) Histone H3 phosphorylation (Ser10, Ser28) and phosphoacetylation (K9S10) are differentially associated with gene expression in liver of rats treated in vivo with acute ethanol. J Pharmacol Exp Ther 340:237-247.

Kaizu T, Ikeda A, Nakao A, Tsung A, Toyokawa H, Ueki S, Geller DA, and Murase N (2008) Protection of transplant-induced hepatic ischemia/reperfusion injury with carbon monoxide via MEK/ERK1/2 pathway downregulation. Am J Physiol Gastrointest Liver Physiol 294:G236-G244.

Kim JS and Shukla SD (2006) Acute in vivo effect of ethanol (binge drinking) on histone H3 modifications in rat tissues. Alcohol Alcohol 41:126-132.

Kirpich I, Ghare S, Zhang J, Gobejishvili L, Kharebava G, Barve SJ, Barker D, Moghe A, McClain CJ, and Barve S (2012) Binge alcohol-induced microvesicular liver steatosis and injury are associated with down-regulation of hepatic Hdac 1, 7, 9, 10, 11 and up-regulation of Hdac 3. Alcohol Clin Exp Res 36:1578-1586.

Kirpich I and McClain C (2017) More alcohol, more liver injury: not always true. Alcohol Alcohol 52:627-628.

Kirpich I, Zhang J, Gobejishvili L, Kharebava G, Barker D, Ghare S, Joshi-Barve S, McClain CJ, and Barve S (2013) Binge ethanol-induced HDAC3 down-regulates Cpt1 $\alpha$ expression leading to hepatic steatosis and injury. Alcohol Clin Exp Res 37: 1920-1929. 
Kirpich IA, McClain CJ, Vatsalya V, Schwandt M, Phillips M, Falkner KC, Zhang L, Harwell C, George DT, and Umhau JC (2017) Liver injury and endotoxemia in male and female alcohol-dependent individuals admitted to an alcohol treatment program. Alcohol Clin Exp Res 41:747-757.

Kono H, Wheeler MD, Rusyn I, Lin M, Seabra V, Rivera CA, Bradford BU, Forman DT, and Thurman RG (2000) Gender differences in early alcohol-induced liver injury: role of CD14, NF-kappaB, and TNF-alpha. Am J Physiol Gastrointest Liver Physiol 278:G652-G661.

Kourkoumpetis T and Sood G (2019) Pathogenesis of alcoholic liver disease: an update. Clin Liver Dis 23:71-80.

Lee YJ and Shukla SD (2005) Pro- and anti-apoptotic roles of c-Jun N-terminal kinase (JNK) in ethanol and acetaldehyde exposed rat hepatocytes. Eur $J$ Pharmacol 508:31-45.

Mandrekar P and Szabo G (2009) Signalling pathways in alcohol-induced liver inflammation. J Hepatol 50:1258-1266.

Nguyen VA and Gao B (2002) Expression of interferon alfa signaling components in human alcoholic liver disease. Hepatology 35:425-432.

Ohri S, Sharma D, and Dixit A (2002) Modulation of c-myc and c-fos gene expression in regenerating rat liver by 2-mercaptopropionylglycine. Cell Biol Int 26:187-192.

Omodeo SMF, Cestaro B, Mascherpa A, Monti D, and Masserini M (1989) Enzymatic synthesis and thermotropic behaviour of phosphatidylethanol. Chem Phys Lipids 50:135-142.

Park PH, Lim RW, and Shukla SD (2005) Involvement of histone acetyltransferase (HAT) in ethanol-induced acetylation of histone H3 in hepatocytes: potential mechanism for gene expression. Am J Physiol Gastrointest Liver Physiol 289:G1124-G1136.

Puigserver P and Spiegelman BM (2003) Peroxisome proliferator-activated receptorgamma coactivator 1 alpha (PGC-1 alpha): transcriptional coactivator and metabolic regulator. Endocr Rev 24:78-90.

Purow B (2015) Molecular pathways: targeting diacylglycerol kinase alpha in cancer. Clin Cancer Res 21:5008-5012.

Rong X, Wang B, Palladino END, de Aguiar Vallim TQ, Ford DA, and Tontonoz P (2017) ER phospholipid composition modulates lipogenesis during feeding and in obesity. J Clin Invest 127:3640-3651.

Sato N, Lindros KO, Baraona E, Ikejima K, Mezey E, Järveläinen HA and Ramchandani VA (2001) Sex difference in alcohol-related organ injury. Alcoho Clin Exp Res 25 (5 Suppl ISBRA):40S-45S

Schattenberg JM, Wang Y, Rigoli RM, Koop DR, and Czaja MJ (2004) CYP2E1 overexpression alters hepatocyte death from menadione and fatty acids by activation of ERK1/2 signaling. Hepatology 39:444-455.
Shield KD, Soerjomataram I, and Rehm J (2016) Alcohol use and breast cancer: a critical review. Alcohol Clin Exp Res 40:1166-1181.

Shukla SD and Aroor AR (2006) Epigenetic effects of ethanol on liver and gastrointestinal injury. World J Gastroenterol 12:5265-5271.

Shukla SD, Aroor AR, Restrepo R, Kharbanda KK, and Ibdah JA (2015a) In vivo acute on chronic ethanol effects in liver: a mouse model exhibiting exacerbated injury, altered metabolic and epigenetic responses. Biomolecules 5:3280-3294.

Shukla SD, Pruett SB, Szabo G, and Arteel GE (2013a) Binge ethanol and liver: new molecular developments. Alcohol Clin Exp Res 37:550-557.

Shukla SD, Restrepo R, Fish P, Lim RW, and Ibdah JA (2015b) Different mechanisms for histone acetylation by ethanol and its metabolite acetate in rat primary hepatocytes. J Pharmacol Exp Ther 354:18-23.

Shukla SD and Zakhari S (2013b) Epigenetics-new frontier for alcohol research [Editorial]. Alcohol Res 35:1-2.

Taylor SA and Miloh T (2019) Adolescent alcoholic liver disease. Clin Liver Dis 23: $51-54$.

van der Veen JN, Kennelly JP, Wan S, Vance JE, Vance DE, and Jacobs RL (2017) The critical role of phosphatidylcholine and phosphatidylethanolamine metabolism in health and disease. Biochim Biophys Acta Biomembr 1859 (9 Pt B):1558-1572

Venugopal SK, Chen J, Zhang Y, Clemens D, Follenzi A, and Zern MA (2007) Role of MAPK phosphatase-1 in sustained activation of JNK during ethanol-induced apoptosis in hepatocyte-like VL-17A cells. J Biol Chem 282:31900-31908.

Viel G, Boscolo-Berto R, Cecchetto G, Fais P, Nalesso A, and Ferrara SD (2012) Phosphatidylethanol in blood as a marker of chronic alcohol use: a systematic review and meta-analysis. Int J Mol Sci 13:14788-14812.

Yin M, Ikejima K, Wheeler MD, Bradford BU, Seabra V, Forman DT, Sato N, and Thurman RG (2000) Estrogen is involved in early alcohol-induced liver injury in a rat enteral feeding model. Hepatology 31:117-123.

Weigel C, Veldwijk MR, Oakes CC, Siebold P, Slynko A, Liesenfeld DB, Rabionet M, Hanke SA, Wenz F, Sperk E, et al. (2016) Epigenetic regulation of diacylglycerol kinase alpha promotes radiation-induced fibrosis. Nat Commun 7:10893.

White AM, Tapert S, and Shukla SD (2018) Binge drinking. Alcohol Res 39:1-3.

Address correspondence to: Shivendra D. Shukla, Department of Medical Pharmacology and Physiology, University of Missouri School of Medicine, Columbia, MO 65212. E-mail: shuklasd@missouri.edu 